\title{
After the crisis, the meaning of new growth
}

\author{
Pier Carlo Padoan ${ }^{1}$
}

Received: 11 September 2017/Accepted: 18 September 2017/Published online: 23 September 2017

(C) Springer International Publishing AG 2017

The Editor in Chief thanks Prof. Pier Carlo Padoan for giving permission to publish it. The title of the editorial and those of the paragraphs have been chosen by the Editor in Chief.

\section{The consequences of a deep crisis}

It is a great pleasure for me to be here today, to receive your statements and continue a dialogue between the policy community, which I am a pro tempore member, and the scientific community.

Let me start from a point of reflection on the consequences of the crisis. The global economy seems to be exiting the deep crisis, which has generated deep scars and these scars still have to be addressed. But it also has generated a permanent slipover over the policy debate. There is wide spread skepticism among citizens with respect to policy making, policy makers and policy institutions because of their apparent inability to deal with challenges such as poor state of the economy, large unemployment, loss of welfare, increasing inequalities. This state of dissatisfaction feeds on a demand for change.

The problem is that this demand for change takes often the culture of rejection, culture of denial, convictions, beliefs, expectations and opinions are translated into solutions that are hardly based on sound assessment and analysis. This of course has possible negative implications for governments and I would also say for democracy.

This editorial is the transcription with some adjustments of the speech delivered at Accademia Nazionale dei Lincei by the Italian Minister of Economy and Finance at the Conference of the G7 Academies of Science meeting held in Rome on the 3rd of May 2017.

Pier Carlo Padoan

segreteria.ministro@mef.gov.it

1 Ministry of Economy and Finance, Rome, Italy 
Policy makers need the scientific community to provide best efforts to improve the quality of the public debate, about the economy, about healthcare, about the environment, as you are doing now. It is important, I would say imperative, to prevent decision making to be made on uninformed beliefs, emotions and denial.

So again, the contribution you are producing to the G7 debate, of which this year Italy holds the Presidency, is extremely welcomed and timely. Your three statements are all very relevant and on behalf of the Government let me thank you again for this effort, let me thank again the Accademia dei Lincei and Alberto Quadrio-Curzio for his own personal effort.

\section{The three joint statements}

Just a few words about two of the three joint statements. Cultural heritage is part of the identity of each nation, taking care of such a heritage is a duty not only for the sake of the past, but I would say even more importantly because it represents the basis on which we build our future. I am sure that my colleague Dario Franceschini will elaborate more on that when he comes to address this audience. Secondly, as we have just heard, human beings will live longer than they used to in the past. This is of course good news. It also requires that the quality of a longer life is deemed to be satisfactory. As it has already been pointed out, finance ministers cannot ignore the increasing pressure that aging society has put on public finances, in terms of resources certainly, but even more so in terms of the quality of the public approach and public budget utilization to deal with aging societies.

Let me come now briefly to the third item on New economic growth which is something that I will devote more attention, also because of my education as an economist.

\section{The structural challenges}

The global economy is now completing what in the recent Spring Meetings of the IMF and World Bank has been described as the macroeconomic cycle. For the economists among you this translates into the fact that observed or effective growth is approaching potential growth. So, a gap is being closed. This does not mean however that the crisis is behind us, quite the contrary.

Now economic policy makers and policy makers around the world are facing what I would like to call a structural challenge. A structural twin challenge indeed. One, first component of that challenge is: Where is growth coming from? And one of the contributions you have provided offered to the debate deals exactly with that issue and strikes exactly the right point-it's about technology. We have been witnessing over the recent past declining productivity growth almost everywhere, especially in the advanced economies. This trend has to be inverted, this trend can be inverted.

I am one of those who believe that technology gains are still to be extracted out of the unknown, about knowledge, about research. So we have a huge potential for 
growth enhancing measures before us, which are very nicely and effectively summarized in one of your documents. So, more productivity related to more innovation, more knowledge, more human capital application.

However, there is a part of the literature that points out to a negative implication of that, which can be increasing inequality due to technological change, through a number of channels, one of which may be increasing job displacement and therefore less employment, which is one of the main sources of rising inequalities. So how do we reconcile this? This is one of the big policy challenges that the international policy community is now facing. This has been taken up as an explicit policy target in the G20 and certainly so in the G7.

Indeed, the Italian G7 Presidency for this year has put inclusive growth as one of the main pillars in the agenda. But here is where you have to move from principles to policy action. How do we translate into effective policy action at the G7, and also at the G20 level for an inclusive growth? Inclusive growth is something which allows to exploit at best innovation and technological change, but also includes increasing parts of society to exploit the benefits in terms of higher employment, better quality of jobs, better lifestyle. Is this a common recipe? It is, I would say, a common principle. It is much more difficult to say there is a common recipe.

Indeed, one third feature of the global perspective, the global economy and society is that you are witnessing differences across the globe in terms of policy lifestyles, policy strategies and therefore it is very hard, possibly impossible, to say that there is one recipe to deal with the challenges of technological change, increasing productivity, and at the same time inclusiveness.

\section{The role of policy makers}

So the ambition of policy makers must be put at the appropriate level. It must be forward looking enough. It must be however also realistic enough to say that something can be achieved in the relevant time scale, otherwise it's just exciting discussion, but not translated into how our lifestyle has changed. So this is the approach we are taking at the G7 level to deal with what can be done at the policy level in terms of inclusive growth. First of all, it must be recognized that there are national specificities to deal with inclusive growth. In some countries, this takes the form of high youth unemployment. In other countries, the women participation to the labour force is very limited and difficult, to say the least. In other countries because of extreme poverty, in other countries again because of regional divides and of course this is also one of the features of my country. In each case there is a common element, but there is also different policy implications.

For the policy makers this has one obvious consequence, that there is no shortcut to deal with inclusive growth. Indeed, inclusive growth is a complex outcome of a hopefully careful and cleaver use of all the policy instruments that are available and indeed, including in my own personal experience, what the policy maker is facing is at the same time an increasing complexity in the policy problem, but also an increasing richness in the policy tool kit. A duty of the policy maker is to try to 
exploit that tool kit at its best. This is why again the quality of the policy debate which the scientific community can powerfully influence is so important.

We need to come up with innovative solutions in the use of policy making tools and just let me go towards the conclusion of my short remarks by saying that this is what certainly this government has been trying to do. To use at the same time budget policy, I am not saying fiscal policy, I am saying budget policy because what matters is the size of the public budget yes, but even more so the composition of the public budget in terms of where to put resources when you decide spending, and where to extract resources when you decide about revenue sources and you can do a lot in terms of reallocation of resources to the budget with a given amount of available financial resources for the state and of course Italy is one country where the public finance constraints is very relevant and very heavy.

The second policy agenda is structural, there is a huge amount of things that can be done combining appropriately structured incentives in changing the way firms, individuals, families, and institutions behave and therefore generate effects and consequences on welfare. We need to understand even better how structural change can affect the way we use resources and the way we impact on productivity and inequality. The themes you have chosen to deal with, healthcare, but also technology, and of course cultural heritage, are all areas where we need to understand better how the systems of healthcare, the systems of cultural heritage, of course how the economic systems operate so that we can impact the way they generate outcome.

Needless to say, one element which is common to all of these items and possibly others is education. Education is key to the quantity and quality of future welfare and the way we shape education, the way the scientific community contributes to shaping education is extremely important and extremely difficult, exactly because we live in a fast changing world. The decision to say the obvious is to decide today what kind of skills availability will be needed and appropriate say two decades from now. This is a very difficult task, but I believe that the scientific community can provide better solutions and continues to provide better solutions, whatever the field education is applied to and of course this involves all policy fields.

\section{The International, European and Italian scenarios}

One final point on the international dimension. I have been hinted at the fact that national specificities are a permanent feature of policy making. Therefore, it is sometimes difficult to identify common policies. However, this is another challenge, which certainly the policy making community must address, which is 'what can we do together?' Can we actually cooperate at the global or international level in some policy areas? This is extremely difficult and especially now with new policy attitudes in key countries emerging, cooperation seems to be very difficult to achieve. Yet, I would like to send a more reassuring message that there are areas in which cooperation is actually increasing.

Europe for instance is one area where there is more cooperation than so often believed. One area which has witnessed a lot of increasing cooperation in practice 
and is already yielding important results is tax policy cooperation, in which the degree of international application of common rules and implementation has made huge progress over the recent past. But this is just an example.

At the same time, and this is my final point, to go back to the issue of using your policy tool kit the best way you can, there are practical ways in which the economic policy paradigm at the national level can be improved to exploit the richness of policy tool kits. This however again requires sound scientific assessment to be applied and here let me just mention one consequence of the financial crisis which broke out almost 10 years ago.

You may recall that when the financial crisis broke out and the dimension and extension of the crisis was finally perceived as being extremely large, deep and devastating, one reaction coming from the scientific community, especially in the economic and social domain, was to say 'we have produced a crisis also because we have looked only at one dimension of economic progress, that is GDP growth'. We need to enrich the set of dimensions measuring progress by adding to GDP other variables and this was by the way fostered by the so called Stiglitz-Sen-Fitoussi report, which put on the table a number of suggestions.

Let me finish with one element of, if I may, national pride. Italy has recently introduced in its official policy making tool kit, in its official Document of Economy and Finance, the beginning of an approach to introduce policy indicators which, in addition to GDP, take into account inequality, sustainability and welfare. This is just beginning to be a challenge. The idea is that we have to link targets in terms of, as I said, inclusiveness, sustainability and welfare, policy tools so that we can measure ex-post whether or not we are making progress.

This is just a little tiny bit of a long journey towards improving the quality of policy making and I do not claim that Italy will always be at the forefront, but I am proud to say that we are the first advanced economy that has introduced officially targets in addition to GDP and public finance, which of course are very much indeed concerns, daily concerns of public finance and finance ministers.

\section{Academies and governments}

With this, I would like to thank you once again for this extremely important contribution. I reiterate the fact that this is not something that ends here, this is something that the government will do its best to incorporate in reflections and hopefully incorporate in policy prescriptions that make lives a better outcome, thank you again. 